\title{
Results on the growth of meromorphic solutions of some linear difference equations with meromorphic coefficients
}

\section{Zhang Li Yuan and Qiu Ling*}

"Correspondence:

qiuling1978@bjut.edu.cn

College of Applied Science, Beijing

University of Technology, Beijing,

100124, China

\begin{abstract}
In this paper, we investigate the growth of meromorphic solutions of some linear difference equations. We obtain some new results on the growth of meromorphic solutions when most coefficients in such equations are meromorphic functions, which are supplements of previous results due to Li and Chen (Adv. Differ. Equ. 2012:203, 2012) and Liu and Mao (Adv. Differ. Equ. 2013:133, 2013).
\end{abstract}

MSC: $30 \mathrm{D} 35 ; 39 \mathrm{~A} 10$

Keywords: complex difference equation; meromorphic coefficients; growth

\section{Introduction and main results}

In this article, a meromorphic function always means meromorphic in the whole complex plane $\mathbb{C}$, and $c$ always means a nonzero constant. We adopt the standard notations of the Nevanlinna value distribution theory of meromorphic functions such as $T(r, f), m(r, f)$ and $N(r, f)$ as explained in [1-3]. In addition, we will use notations $\rho(f)$ to denote the order of growth of a meromorphic function $f(z), \lambda(f)$ to denote the exponents of convergence of the zero sequence of a meromorphic function $f(z), \lambda\left(\frac{1}{f}\right)$ to denote the exponents of convergence of the pole sequence of a meromorphic function $f(z)$, and we define them as follows:

$$
\begin{aligned}
& \rho(f)=\limsup _{r \rightarrow \infty} \frac{\log T(r, f)}{\log r}, \\
& \lambda(f)=\liminf _{r \rightarrow \infty} \frac{\log N\left(r, \frac{1}{f}\right)}{\log r}, \\
& \lambda\left(\frac{1}{f}\right)=\liminf _{r \rightarrow \infty} \frac{\log N(r, f)}{\log r} .
\end{aligned}
$$

Recently, meromorphic solutions of complex difference equations have become a subject of great interest from the viewpoint of Nevanlinna theory due to the apparent role of the existence of such solutions of finite order for the integrability of discrete difference equations.

About the growth of meromorphic solutions of some linear difference equations, some results can be found in [4-20]. Laine and Yang [12] considered the entire functions coefficients case and got the following.

O2014 Yuan and Ling; licensee Springer. This is an Open Access article distributed under the terms of the Creative Commons Attribution License (http://creativecommons.org/licenses/by/2.0), which permits unrestricted use, distribution, and reproduction in any medium, provided the original work is properly cited. 
Theorem A [12] Let $A_{j}(z)(\not \equiv 0)(j=0,1, \ldots, n)$ be entire functions of finite order such that among those coefficients having the maximal order $\rho:=\max _{0 \leq j \leq n} \rho\left(A_{j}\right)$, exactly one has its type strictly greater than the others. Then, for any meromorphic solution $f(z)$ to

$$
A_{n}(z) f(z+n)+\cdots+A_{1}(z) f(z+1)+A_{0}(z) f(z)=0,
$$

we have $\rho(f) \geq \rho+1$.

Chiang and Feng $[13,14]$ improved Theorem A as follows.

Theorem $\mathbf{B}[13,14]$ Let $A_{j}(z)(\not \equiv 0)(j=0,1, \ldots, n)$ be entire functions such that there exists an integer $l, 0 \leq l \leq n$, such that

$$
\rho\left(A_{l}\right)>\max _{0 \leq j \leq n, j \neq l} \rho\left(A_{j}\right)
$$

Suppose that $f(z)$ is a meromorphic solution to

$$
A_{n}(z) f(z+n)+\cdots+A_{1}(z) f(z+1)+A_{0}(z) f(z)=0,
$$

then we have $\rho(f) \geq \rho\left(A_{l}\right)+1$.

Recently in [15], Peng and Chen investigated the order and the hyper-order of solutions of some second-order linear differential equations and proved the following results.

Theorem C [15] Suppose that $A_{j}(z)(\not \equiv 0)(j=1,2)$ are entire functions and $\rho\left(A_{j}\right)<1$. Let $\alpha_{1}, \alpha_{2}$ be two distinct complex numbers such that $\alpha_{1} \alpha_{2} \neq 0$ (suppose that $\left|\alpha_{1}\right| \leq\left|\alpha_{2}\right|$ ). If $\arg \alpha_{1} \neq \pi$ or $\alpha_{1}<-1$, then every solution $f(z) \not \equiv 0$ of the equation

$$
f^{\prime \prime}+e^{-z} f^{\prime}+\left(A_{1} e^{\alpha_{1} z}+A_{2} e^{\alpha_{2} z}\right) f=0
$$

has infinite order and $\rho_{2}(f)=1$.

Moreover, $\mathrm{Xu}$ and Zhang [16] extended the above result from entire coefficients to meromorphic coefficients.

It is well known that $\Delta f(z)=f(z+c)-f(z)$ is regarded as the difference counterpart of $f^{\prime}$. Thus a natural question is: Can we change the above second-order linear differential equation to the linear difference equation? What conditions will guarantee that every meromorphic solution will have infinite order when most coefficients in such equations are meromorphic functions?

Li and Chen [17] considered the following difference equation and obtained the following theorem.

Theorem D [17] Let $k$ be a positive integer, $p$ be a nonzero real number and $f(z)$ be a nonconstant meromorphic solution of the difference equation

$$
\begin{aligned}
& A_{n}(z) f(z+n)+\cdots+A_{2}(z) f(z+2)+\left(A_{1}(z) e^{-p z^{k}}+B_{1}(z)\right) f(z+1) \\
& \quad+\left(A_{0}(z) e^{p z^{k}}+B_{0}(z)\right) f(z)=0,
\end{aligned}
$$


where $A_{j}(z), B_{0}(z), B_{1}(z)(\not \equiv 0)(j=0,1, \ldots, n)$ are all entire functions and $\max \left\{\rho\left(A_{j}\right), \rho\left(B_{0}\right)\right.$, $\left.\rho\left(B_{1}\right)\right\}=\sigma<k$, then we have $\rho(f) \geq k+1$.

The main purpose of this paper is to investigate the growth of meromorphic solutions of certain linear difference equations with meromorphic coefficients. The remainder of the paper studies the properties of meromorphic solutions of a nonhomogeneous linear difference equation. In fact, we prove the following results, in which there are still some coefficients dominating in some angles.

Theorem 1.1 Let $k$ be a positive integer. Suppose that $A_{j}(z), B_{0}(z)(\not \equiv 0)(j=0,1, \ldots, n)$ are all entire functions and $\max \left\{\rho\left(A_{j}\right), \rho\left(B_{0}\right): 0 \leq j \leq n\right\}=\alpha<k$. Let $\alpha_{0}, \beta_{0}$ be two distinct complex numbers such that $\alpha_{0} \beta_{0} \neq 0$ (suppose that $\left|\alpha_{0}\right| \leq\left|\beta_{0}\right|$ ). Let $\alpha_{1}$ be a strictly negative real constant. If $\arg \alpha_{0} \neq \pi$ or $\alpha_{0}<\alpha_{1}$, then every meromorphic solution $f(z) \not \equiv 0$ of the equation

$$
\begin{aligned}
& A_{n}(z) f(z+n)+\cdots+A_{2}(z) f(z+2)+A_{1}(z) e^{\alpha_{1} z^{k}} f(z+1) \\
& +\left(A_{0}(z) e^{\alpha_{0} z^{k}}+B_{0}(z) e^{\beta_{0} z^{k}}\right) f(z)=0
\end{aligned}
$$

satisfies $\rho(f) \geq k+1$.

Theorem 1.2 Suppose that $A_{j}(z), B_{0}(z)(\not \equiv 0)(j=0,1, \ldots, n)$ are all meromorphic functions and $\max \left\{\rho\left(A_{j}\right), \rho\left(B_{0}\right): 0 \leq j \leq n\right\}=\alpha<1$. Let $\alpha_{0}, \beta_{0}$ be two distinct complex numbers such that $\alpha_{0} \beta_{0} \neq 0$ (suppose that $\left|\alpha_{0}\right| \leq\left|\beta_{0}\right|$ ). Let $\alpha_{1}$ be a strictly negative real constant. If $\arg \alpha_{0} \neq$ $\pi$ or $\alpha_{0}<\alpha_{1}$, then every meromorphic solution $f(z) \not \equiv 0$ of the equation

$$
\begin{aligned}
& A_{n}(z) f(z+n)+\cdots+A_{2}(z) f(z+2)+A_{1}(z) e^{\alpha_{1} z} f(z+1) \\
& \quad+\left(A_{0}(z) e^{\alpha_{0} z}+B_{0}(z) e^{\beta_{0} z}\right) f(z)=0
\end{aligned}
$$

satisfies $\rho(f) \geq 2$.

Theorem 1.3 Under the assumption for the coefficients of (1.1) in Theorem 1.1, iff $(z)$ is a finite order meromorphic solution to (1.1), then $\lambda(f-z)=\rho(f)$. What is more, either $k+1 \leq$ $\rho(f) \leq \max \left\{\lambda(f), \lambda\left(\frac{1}{f}\right)\right\}+1$ or $\rho(f)=k+1>\max \left\{\lambda(f), \lambda\left(\frac{1}{f}\right)\right\}+1$.

Theorem 1.4 Under the assumption for the coefficients of (1.2) in Theorem 1.2, iff $(z)$ is a finite order meromorphic solution to (1.2), then $\lambda(f-z)=\rho(f)$. What is more, either $2 \leq$ $\rho(f) \leq \max \left\{\lambda(f), \lambda\left(\frac{1}{f}\right)\right\}+1$ or $\rho(f)=2>\max \left\{\lambda(f), \lambda\left(\frac{1}{f}\right)\right\}+1$.

Liu and Mao [18] considered the meromorphic solutions of the difference equation

$$
a_{k}(z) f(z+k)+\cdots+a_{1}(z) f(z+1)+a_{0}(z) f(z)=0,
$$

one of their results can be stated as follows.

Theorem E [18] Let $a_{j}(z)=A_{j}(z) e^{P_{j}(z)}(j=0,1, \ldots, k)$, where $P_{j}(z)=\alpha_{j n} z^{n}+\cdots+\alpha_{j 0}$ are polynomials with degree $n(\geq 1), A_{j}(z)(\not \equiv 0)$ are entire functions of $\rho\left(A_{j}\right)<n$. If $\alpha_{j n}(j=$ 
$1,2, \ldots, k)$ are distinct complex numbers, then every meromorphic solution $f(\not \equiv 0)$ of Eq. (1.3) satisfies $\rho(f) \geq \max _{0 \leq j \leq k}\left\{\rho\left(a_{j}\right)\right\}+1$.

In this paper, we extend and improve the above result from entire coefficients to meromorphic coefficients in the case where the polynomials $P_{j}(z)$ are of degree 1.

Theorem 1.5 Let $a_{j}(z)=A_{j}(z) e^{\alpha_{j} z}(j=0,1, \ldots, n), \alpha_{j}$ are distinct complex constants, suppose that $A_{j}(z)(\not \equiv 0)$ are meromorphic functions and $\rho\left(A_{j}\right)=\alpha<1$, then every meromorphic solution $f(\not \equiv 0)$ of the equation

$$
a_{n}(z) f(z+n)+\cdots+a_{1}(z) f(z+1)+a_{0}(z) f(z)=0
$$

satisfies $\rho(f) \geq 2$.

Theorem 1.6 Let $a_{j}(z)=A_{j}(z) e^{\alpha_{j} z}+D_{j}(z)(j=0,1, \ldots, n)$, $\alpha_{j}$ are distinct complex constants, suppose that $A_{j}(z)(\not \equiv 0), D_{j}(z)(\not \equiv 0)$ are meromorphic functions and $\rho\left(A_{j}\right)=\alpha<1, \rho\left(D_{j}\right)=$ $\beta<1$, then every meromorphic solution $f(\not \equiv 0)$ of Eq. (1.4) satisfies $\rho(f) \geq 2$.

Next we consider the properties of meromorphic solutions of the nonhomogeneous linear difference equation corresponding to (1.4)

$$
a_{n}(z) f(z+n)+\cdots+a_{1}(z) f(z+1)+a_{0}(z) f(z)=F(z)
$$

where $F(z)(\not \equiv 0)$ is a meromorphic function.

Theorem 1.7 Let $a_{j}(z)(j=0,1, \ldots, n)$ satisfy the hypothesis of Theorem 1.5 or Theorem 1.6, and let $F(z)$ be a meromorphic function of $\rho(F)<1$, then at most one meromorphic solution $f_{0}$ of Eq. (1.5) satisfies $1 \leq \rho\left(f_{0}\right) \leq 2$ and $\max \left\{\lambda\left(f_{0}\right), \lambda\left(\frac{1}{f_{0}}\right)\right\}=\rho\left(f_{0}\right)$, the other solutions $f$ satisfy $\rho(f) \geq 2$.

\section{Some lemmas}

In this section, we present some lemmas which will be needed in the sequel.

Lemma 2.1 [13] Let $f(z)$ be a meromorphic function of finite order $\rho, \epsilon$ be a positive constant, $\eta_{1}$ and $\eta_{2}$ be two distinct nonzero complex constants. Then

$$
m\left(r, \frac{f\left(z+\eta_{1}\right)}{f\left(z+\eta_{2}\right)}\right)=O\left(r^{\rho-1+\epsilon}\right),
$$

and there exists a subset $E_{1} \subset(1, \infty)$ of finite logarithmic measure such that, for all $z$ satisfying $|z|=r \notin[0,1] \cup E_{1}$, and as $r \rightarrow \infty$,

$$
\exp \left\{-r^{\rho-1+\epsilon}\right\} \leq\left|\frac{f\left(z+\eta_{1}\right)}{f\left(z+\eta_{2}\right)}\right| \leq \exp \left\{r^{\rho-1+\epsilon}\right\} .
$$

Lemma 2.2 [19] Let $f(z)$ be a meromorphic function of finite order $\rho$, then, for any given $\epsilon>0$, there exists a set $E_{2} \subset(1, \infty)$ offinite logarithmic measure such that, for all $z$ satisfying $|z|=r \notin[0,1] \cup E_{2}$, and as $r \rightarrow \infty$,

$$
\exp \left\{-r^{\rho+\epsilon}\right\} \leq|f(z)| \leq \exp \left\{r^{\rho+\epsilon}\right\}
$$


Lemma $2.3[20]$ Suppose that $P(z)=(\alpha+i \beta) z^{n}+\cdots(\alpha, \beta$ are real numbers, $|\alpha|+|\beta| \neq 0)$ is a polynomial with degree $n \geq 1, A(z)(\not \equiv 0)$ is an entire function with $\rho(A)<n$. Set $g(z)=$ $A(z) e^{P(z)}, z=r e^{i \theta}, \delta(P, \theta)=\alpha \cos n \theta-\beta \sin n \theta$. Then, for any given $\epsilon>0$, there exists a set $E_{3} \subset[0,2 \pi)$ that has linear measure zero such that for any $\theta \in[0,2 \pi) \backslash\left(E_{3} \cup E_{4}\right)$, there is $R>0$ such that for $|z|=r>R$, we have

(i) if $\delta(P, \theta)>0$, then

$$
\exp \left\{(1-\epsilon) \delta(P, \theta) r^{n}\right\}<\left|g\left(r e^{i \theta}\right)\right|<\exp \left\{(1+\epsilon) \delta(P, \theta) r^{n}\right\}
$$

(ii) if $\delta(P, \theta)<0$, then

$$
\exp \left\{(1+\epsilon) \delta(P, \theta) r^{n}\right\}<\left|g\left(r e^{i \theta}\right)\right|<\exp \left\{(1-\epsilon) \delta(P, \theta) r^{n}\right\},
$$

where $E_{4}=\{\theta \in[0,2 \pi): \delta(P, \theta)=0\}$ is a finite set.

Lemma 2.3 applies in Theorem 1.1 where $A(z)(\not \equiv 0)$ is an entire function.

Lemma 2.4 [15] Suppose that $n \geq 1$ is a positive integer. Let $P_{j}(z)=a_{j n} z^{n}+\cdots(j=1,2)$ be nonconstant polynomials, where $a_{j q}(q=1, \ldots, n)$ are distinct complex numbers and $a_{1 n} a_{2 n} \neq 0$. Set $z=r e^{i \theta}, a_{j n}=\left|a_{j n}\right| e^{i \theta_{j}}, \theta_{j} \in\left[-\frac{\pi}{2}, \frac{3 \pi}{2}\right), \delta\left(P_{j}, \theta\right)=\left|a_{j n}\right| \cos \left(\theta_{j}+n \theta\right)$, then there is a set $E_{5} \subset\left[-\frac{\pi}{2 n}, \frac{3 \pi}{2 n}\right)$ that has linear measure zero. If $\theta_{1} \neq \theta_{2}$, then there exists a ray $\arg z=\theta$, $\theta \in\left[-\frac{\pi}{2 n}, \frac{\pi}{2 n}\right) \backslash\left(E_{5} \cup E_{6}\right)$ such that

$$
\delta\left(P_{1}, \theta\right)>0, \quad \delta\left(P_{2}, \theta\right)<0
$$

or

$$
\delta\left(P_{1}, \theta\right)<0, \quad \delta\left(P_{2}, \theta\right)>0
$$

where $E_{6}=\left\{\theta \in\left[-\frac{\pi}{2 n}, \frac{3 \pi}{2 n}\right): \delta\left(P_{j}, \theta\right)=0\right\}$ is a finite set, which has linear measure zero.

In Lemma 2.4, if $\theta \in\left[-\frac{\pi}{2 n}, \frac{\pi}{2 n}\right) \backslash\left(E_{5} \cup E_{6}\right)$ is replaced by $\theta \in\left[\frac{\pi}{2 n}, \frac{3 \pi}{2 n}\right) \backslash\left(E_{5} \cup E_{6}\right)$, then we have the same result.

Lemma 2.5 [19] Consider $g(z)=A(z) e^{a z}$, where $A(z)(\not \equiv 0)$ is a meromorphic function with $\rho(A)=\alpha<1, a$ is a complex constant, $a=|a| e^{i \varphi}(\varphi \in[0,2 \pi))$. Set $E_{7}=\{\theta \in[0,2 \pi): \cos (\varphi+$ $\theta)=0\}$, then $E_{7}$ is a finite set. Then, for any given $\epsilon(0<\epsilon<1-\alpha)$, there exists a set $E_{8} \subset$ $[0,2 \pi)$ that has linear measure zero, if $z=r e^{i \theta}, \theta \in[0,2 \pi) \backslash\left(E_{7} \cup E_{8}\right)$, then we have (when $r$ is sufficiently large):

(i) if $\delta(a z, \theta)>0$, then

$$
\exp \{(1-\epsilon) \delta(a z, \theta) r\} \leq\left|g\left(r e^{i \theta}\right)\right| \leq \exp \{(1+\epsilon) \delta(a z, \theta) r\}
$$

(ii) if $\delta(a z, \theta)<0$, then

$$
\exp \{(1+\epsilon) \delta(a z, \theta) r\} \leq\left|g\left(r e^{i \theta}\right)\right| \leq \exp \{(1-\epsilon) \delta(a z, \theta) r\},
$$

where $\delta(a z, \theta)=|a| \cos (\varphi+\theta)$. 
Lemma 2.5 applies in Theorem 1.2 where $A(z)(\not \equiv 0)$ is a meromorphic function.

Lemma 2.6 [3] Let $f_{j}(z)(j=1,2, \ldots, n, n \geq 2)$ be meromorphic functions and $g_{j}(z)(j=$ $1,2, \ldots, n, n \geq 2)$ be entire functions such that

(i) $\sum_{j=1}^{n} f_{j}(z) e^{g_{j}(z)} \equiv 0$,

(ii) $g_{j}(z)-g_{k}(z)$ are not constant functions for $1 \leq j<k \leq n$,

(iii) $T\left(r, f_{j}\right)=o\left(T\left(r, e^{g_{h}-g_{k}}\right)\right)(r \rightarrow \infty, r \notin E)$, where $E$ is an exceptional set of finite linear measure, $1 \leq j \leq n$ and $1 \leq h<k \leq n$.

Then $f_{j}(z) \equiv 0(j=1,2, \ldots, n)$.

Lemma $2.7[18]$ Let $G(z)=\sum_{j=1}^{k} B_{j}(z) e^{P_{j}(z)}$, where $P_{j}(z)=\alpha_{j n} z^{n}+\cdots+\alpha_{j 0}$ are polynomials with degree $n(\geq 1), B_{j}(\not \equiv 0)$ are meromorphic functions of order $\rho\left(B_{j}\right)<n$. If $\alpha_{j n}$ $(j=1,2, \ldots, k)$ are distinct complex numbers, then $\rho(G)=n$.

\section{Proofs of the results}

\subsection{The proof of Theorem 1.1}

Suppose that (1.1) admits a nontrivial meromorphic solution $f(z)$ such that $\rho(f)<k+1$, then by Lemma 2.1, for any given $\epsilon$ such that $0<\epsilon<\min \left\{k+1-\rho, k-\alpha, \frac{\left|\beta_{0}\right|-\left|\alpha_{0}\right|}{\left|\beta_{0}\right|+\left|\alpha_{0}\right|}\right\}$, we have

$$
\exp \left\{-r^{\rho(f)-1+\epsilon}\right\} \leq\left|\frac{f(z+j)}{f(z)}\right| \leq \exp \left\{r^{\rho(f)-1+\epsilon}\right\}, \quad j=0, \ldots, n,
$$

for all $r$ outside of a possible exceptional set $E_{1}$ with finite logarithmic measure.

Applying Lemma 2.2, we have

$$
\begin{aligned}
& \exp \left\{-r^{\alpha+\epsilon}\right\} \leq\left|A_{j}(z)\right| \leq \exp \left\{r^{\alpha+\epsilon}\right\}, \quad j=0, \ldots, n, \\
& \exp \left\{-r^{\alpha+\epsilon}\right\} \leq\left|B_{0}(z)\right| \leq \exp \left\{r^{\alpha+\epsilon}\right\},
\end{aligned}
$$

for all $r$ outside of a possible exceptional set $E_{2}$ with finite linear measure.

Therefore from (1.1) we can get that

$$
\frac{A_{n}(z) f(z+n)}{f(z)}+\cdots+\frac{A_{2}(z) f(z+2)}{f(z)}+\frac{A_{1}(z) e^{\alpha_{1} z^{k}} f(z+1)}{f(z)}+A_{0}(z) e^{\alpha_{0} z^{k}}+B_{0}(z) e^{\beta_{0} z^{k}}=0,
$$

i.e.,

$$
\begin{aligned}
& \left|A_{0}(z) e^{\alpha_{0} z^{k}}+B_{0}(z) e^{\beta_{0} z^{k}}\right| \\
& \quad \leq\left|\frac{A_{n}(z) f(z+n)}{f(z)}\right|+\cdots+\left|\frac{A_{2}(z) f(z+2)}{f(z)}\right|+\left|\frac{A_{1}(z) e^{\alpha_{1} z^{k}} f(z+1)}{f(z)}\right| .
\end{aligned}
$$

Setting $\alpha_{0}=\left|\alpha_{0}\right| e^{i \theta_{0}}, \beta_{0}=\left|\beta_{0}\right| e^{i \varphi_{0}}\left(\theta_{0}, \varphi_{0} \in\left[-\frac{\pi}{2}, \frac{3 \pi}{2}\right)\right)$.

Case 1. $\arg \alpha_{0} \neq \pi$, which is $\theta_{0} \neq \pi$.

Subcase 1.1. Assume that $\theta_{0} \neq \varphi_{0}$. By Lemma 2.4, for the above $\epsilon$, there is a ray $\arg z=\theta$ such that $\theta \in\left[-\frac{\pi}{2 k}, \frac{\pi}{2 k}\right) \backslash\left(E_{1} \cup E_{2} \cup E_{5} \cup E_{6}\right)$ (where $E_{5}$ and $E_{6}$ are defined as in Lemma 2.4, $E_{1} \cup E_{2} \cup E_{5} \cup E_{6}$ is of linear measure zero) satisfying $\delta\left(\alpha_{0} z^{k}, \theta\right)>0, \delta\left(\beta_{0} z^{k}, \theta\right)<0$ or $\delta\left(\alpha_{0} z^{k}, \theta\right)<0, \delta\left(\beta_{0} z^{k}, \theta\right)>0$ for a sufficiently large $r$. 
Since $A_{j}(z), B_{0}(z)(\not \equiv 0)(j=0,1, \ldots, n)$ are entire functions and $\max \left\{\rho\left(A_{j}\right), \rho\left(B_{0}\right): 0 \leq j \leq\right.$ $n\}=\alpha<k$, then when $\delta\left(\alpha_{0} z^{k}, \theta\right)>0, \delta\left(\beta_{0} z^{k}, \theta\right)<0$ for a sufficiently large $r$, by Lemma 2.3, we have

$$
\begin{aligned}
& \left|A_{0}(z) e^{\alpha_{0} z^{k}}\right|>\exp \left\{(1-\epsilon) \delta\left(\alpha_{0} z^{k}, \theta\right) r^{k}\right\}, \\
& \left|B_{0}(z) e^{\beta_{0} z^{k}}\right|<\exp \left\{(1-\epsilon) \delta\left(\beta_{0} z^{k}, \theta\right) r^{k}\right\}<1 .
\end{aligned}
$$

By (3.5) and (3.6), we have

$$
\begin{aligned}
\left|A_{0}(z) e^{\alpha_{0} z^{k}}+B_{0}(z) e^{\beta_{0} z^{k}}\right| & \geq\left|A_{0}(z) e^{\alpha_{0} z^{k}}\right|-\left|B_{0}(z) e^{\beta_{0} z^{k}}\right| \\
& >\exp \left\{(1-\epsilon) \delta\left(\alpha_{0} z^{k}, \theta\right) r^{k}\right\}-1 \\
& =(1-o(1)) \exp \left\{(1-\varepsilon) \delta\left(\alpha_{0} z^{k}, \theta\right) r^{k}\right\} .
\end{aligned}
$$

Since $\theta \in\left[-\frac{\pi}{2 k}, \frac{\pi}{2 k}\right) \backslash\left(E_{1} \cup E_{2} \cup E_{5} \cup E_{6}\right)$, we know that $\cos k \theta>0$, then $\left|e^{\alpha_{1} z^{k}}\right|=$ $e^{-\left|\alpha_{1}\right| r^{k} \cos k \theta}<1$. Therefore, by (3.2) we obtain

$$
\left|A_{1}(z) e^{\alpha_{1} z^{k}}\right| \leq \exp \left\{r^{\alpha+\epsilon}\right\}
$$

Substituting (3.1), (3.2), (3.3), (3.7) and (3.8) into (3.4), we obtain

$$
(1-o(1)) \exp \left\{(1-\epsilon) \delta\left(\alpha_{0} z^{k}, \theta\right) r^{k}\right\}<n \exp \left\{r^{\alpha+\epsilon}+r^{\rho(f)-1+\epsilon}\right\}
$$

By $\delta\left(\alpha_{0} z^{k}, \theta\right)>0, \alpha+\epsilon<k$ and $\rho(f)-1+\epsilon<k$, we know that (3.9) is a contradiction.

When $\delta\left(\alpha_{0} z^{k}, \theta\right)<0, \delta\left(\beta_{0} z^{k}, \theta\right)>0$, using a proof similar to the above, we can get a contradiction.

Subcase 1.2. Assume that $\theta_{0}=\varphi_{0}$. By Lemma 2.4, for the above $\epsilon$, there is a ray $\arg z=\theta$ such that $\theta \in\left[-\frac{\pi}{2 k}, \frac{\pi}{2 k}\right) \backslash\left(E_{1} \cup E_{2} \cup E_{5} \cup E_{6}\right)$ (where $E_{5}$ and $E_{6}$ are defined as in Lemma 2.4, $E_{1} \cup E_{2} \cup E_{5} \cup E_{6}$ is of linear measure zero) satisfying $\delta\left(\alpha_{0} z^{k}, \theta\right)>0$.

Since $\left|\alpha_{0}\right| \leq\left|\beta_{0}\right|, \alpha_{0} \neq \beta_{0}$, and $\theta_{0}=\varphi_{0}$, then $\left|\alpha_{0}\right|<\left|\beta_{0}\right|$, thus $\delta\left(\beta_{0} z^{k}, \theta\right)>\delta\left(\alpha_{0} z^{k}, \theta\right)>0$. For a sufficiently large $r$, by Lemma 2.3 , we get

$$
\begin{aligned}
& \left|A_{0}(z) e^{\alpha_{0} z^{k}}\right|<\exp \left\{(1+\epsilon) \delta\left(\alpha_{0} z^{k}, \theta\right) r^{k}\right\}, \\
& \left|B_{0}(z) e^{\beta_{0} z^{k}}\right|>\exp \left\{(1-\epsilon) \delta\left(\beta_{0} z^{k}, \theta\right) r^{k}\right\} .
\end{aligned}
$$

By (3.10) and (3.11), we get

$$
\begin{aligned}
\left|A_{0}(z) e^{\alpha_{0} z^{k}}+B_{0}(z) e^{\beta_{0} z^{k}}\right| & \geq\left|B_{0}(z) e^{\beta_{0} z^{k}}\right|-\left|A_{0}(z) e^{\alpha_{0} z^{k}}\right| \\
& >\exp \left\{(1-\epsilon) \delta\left(\beta_{0} z^{k}, \theta\right) r^{k}\right\}-\exp \left\{(1+\epsilon) \delta\left(\alpha_{0} z^{k}, \theta\right) r^{k}\right\} \\
& =M_{1} \exp \left\{(1+\epsilon) \delta\left(\alpha_{0} z^{k}, \theta\right) r^{k}\right\},
\end{aligned}
$$

where $M_{1}=\exp \left\{\left[(1-\epsilon) \delta\left(\beta_{0} z^{k}, \theta\right)-(1+\epsilon) \delta\left(\alpha_{0} z^{k}, \theta\right)\right] r^{k}\right\}-1$.

Since $0<\epsilon<\min \left\{k+1-\rho, k-\alpha, \frac{\left|\beta_{0}\right|-\left|\alpha_{0}\right|}{\left|\beta_{0}\right|+\left|\alpha_{0}\right|}\right\}$, we see that $(1-\epsilon) \delta\left(\beta_{0} z^{k}, \theta\right)-(1+\epsilon) \delta\left(\alpha_{0} z^{k}, \theta\right)>$ 0 , then $\exp \left\{\left[(1-\epsilon) \delta\left(\beta_{0} z^{k}, \theta\right)-(1+\epsilon) \delta\left(\alpha_{0} z^{k}, \theta\right)\right] r^{k}\right\}>1, M_{1}>0$. 
Since $\theta \in\left[-\frac{\pi}{2 k}, \frac{\pi}{2 k}\right) \backslash\left(E_{1} \cup E_{2} \cup E_{5} \cup E_{6}\right)$, we know that $\cos k \theta>0$, then $\left|e^{\alpha_{1} z^{k}}\right|=$ $e^{-\left|\alpha_{1}\right| r^{k} \cos k \theta}<1$. Therefore, by (3.2) we obtain

$$
\left|A_{1}(z) e^{\alpha_{1} z^{k}}\right| \leq \exp \left\{r^{\alpha+\epsilon}\right\}
$$

Substituting (3.1), (3.2), (3.3), (3.12) and (3.13) into (3.3), we obtain

$$
M_{1} \exp \left\{(1+\epsilon) \delta\left(\alpha_{0} z^{k}, \theta\right) r^{k}\right\} \leq n \exp \left\{r^{\alpha+\epsilon}+r^{\rho(f)-1+\epsilon}\right\}
$$

By $\delta\left(\alpha_{0} z^{k}, \theta\right)>0, \alpha+\epsilon<k$ and $\rho(f)-1+\epsilon<k$, we know that (3.14) is a contradiction.

Case 2. $\alpha_{0}<\alpha_{1}$, which is $\theta_{0}=\pi$.

Subcase 2.1. Assume that $\theta_{0} \neq \varphi_{0}$, then $\varphi_{0} \neq \pi$. By Lemma 2.4, for the above $\epsilon$, there is a ray $\arg z=\theta$ such that $\theta \in\left[-\frac{\pi}{2 k}, \frac{\pi}{2 k}\right) \backslash\left(E_{1} \cup E_{2} \cup E_{5} \cup E_{6}\right.$ ) (where $E_{5}$ and $E_{6}$ are defined as in Lemma 2.4, $E_{1} \cup E_{2} \cup E_{5} \cup E_{6}$ is of linear measure zero) satisfying $\delta\left(\beta_{0} z^{k}, \theta\right)>0$. Since $\cos k \theta>0$, we have $\delta\left(\alpha_{0} z^{k}, \theta\right)=\left|\alpha_{0}\right| \cos \left(\theta_{0}+k \theta\right)=-\left|\alpha_{0}\right| \cos k \theta<0$. For a sufficiently large $r$, by Lemma 2.3, we get

$$
\begin{aligned}
& \left|A_{0}(z) e^{\alpha_{0} z^{k}}\right|<\exp \left\{(1-\epsilon) \delta\left(\alpha_{0} z^{k}, \theta\right) r^{k}\right\}<1, \\
& \left|B_{0}(z) e^{\beta_{0} z^{k}}\right|>\exp \left\{(1-\epsilon) \delta\left(\beta_{0} z^{k}, \theta\right) r^{k}\right\} .
\end{aligned}
$$

By (3.15) and (3.16), we get

$$
\begin{aligned}
\left|A_{0}(z) e^{\alpha_{0} z^{k}}+B_{0}(z) e^{\beta_{0} z^{k}}\right| & \geq\left|B_{0}(z) e^{\beta_{0} z^{k}}\right|-\left|A_{0}(z) e^{\alpha_{0} z^{k}}\right| \\
& >\exp \left\{(1-\epsilon) \delta\left(\beta_{0} z^{k}, \theta\right) r^{k}\right\}-1
\end{aligned}
$$

Using the same reasoning as in Subcase 1.1, we can get a contradiction.

Subcase 2.2. Assume that $\theta_{0}=\varphi_{0}$, then $\theta_{0}=\varphi_{0}=\pi$. By Lemma 2.4, for the above $\epsilon$, there is a ray $\arg z=\theta$ such that $\theta \in\left[\frac{\pi}{2 k}, \frac{3 \pi}{2 k}\right) \backslash\left(E_{1} \cup E_{2} \cup E_{5} \cup E_{6}\right)$ (where $E_{5}$ and $E_{6}$ are defined as in Lemma 2.4, $E_{1} \cup E_{2} \cup E_{5} \cup E_{6}$ is of linear measure zero), then $\cos k \theta<0, \delta\left(\alpha_{0} z^{k}, \theta\right)=$ $\left|\alpha_{0}\right| \cos \left(\theta_{0}+k \theta\right)=-\left|\alpha_{0}\right| \cos k \theta>0, \delta\left(\beta_{0} z^{k}, \theta\right)=\left|\beta_{0}\right| \cos \left(\varphi_{0}+k \theta\right)=-\left|\beta_{0}\right| \cos k \theta>0$.

Since $\left|\alpha_{0}\right| \leq\left|\beta_{0}\right|, \alpha_{0} \neq \beta_{0}$, and $\theta_{0}=\varphi_{0}$, then $\left|\alpha_{0}\right|<\left|\beta_{0}\right|$, thus $\delta\left(\beta_{0} z^{k}, \theta\right)>\delta\left(\alpha_{0} z^{k}, \theta\right)>0$. For a sufficiently large $r$, we get (3.10), (3.11) and (3.12) hold.

Using the same reasoning as in Subcase 1.2, we can get a contradiction. Thus we have $\rho(f) \geq k+1$.

\subsection{The proof of Theorem 1.2}

Since $A_{j}(z), B_{0}(z)(\not \equiv 0)(j=0,1, \ldots, n)$ are meromorphic functions and $\max \left\{\rho\left(A_{j}\right), \rho\left(B_{0}\right)\right.$ : $0 \leq j \leq n\}=\alpha<1$, then when $\delta\left(\alpha_{0} z, \theta\right)>0, \beta\left(a_{0} z, \theta\right)<0$ for a sufficiently large $r$, by Lemma 2.5, we have

$$
\begin{aligned}
& \left|A_{0}(z) e^{\alpha_{0} z}\right| \geq \exp \left\{(1-\epsilon) \delta\left(\alpha_{0} z, \theta\right) r\right\}, \\
& \left|B_{0}(z) e^{\beta_{0} z}\right| \leq \exp \left\{(1-\epsilon) \delta\left(\beta_{0} z, \theta\right) r\right\}<1 .
\end{aligned}
$$

Then, using a similar argument to that of Theorem 1.1, we obtain a contradiction. 


\subsection{The proof of Theorems 1.3 and 1.4}

Suppose that $f(z)$ is a nonconstant meromorphic solution of (1.1) such that $\rho(f)<\infty$. We first prove that $\lambda(f-z)=\rho(f)$. Submitting $f(z)=g(z)+z$ into (1.1), we get

$$
\begin{gathered}
A_{n}(z) g(z+n)+\cdots+A_{2}(z) g(z+2)+A_{1}(z) e^{\alpha_{1} z^{k}} g(z+1) \\
+\left(A_{0}(z) e^{\alpha_{0} z^{k}}+B_{0}(z) e^{\beta_{0} z^{k}}\right) g(z)=D(z),
\end{gathered}
$$

where

$$
\begin{aligned}
D(z)= & -z\left\{A_{n}(z)+\cdots+A_{2}(z)+A_{1}(z) e^{\alpha_{1} z^{k}}+\left(A_{0}(z) e^{\alpha_{0} z^{k}}+B_{0}(z) e^{\beta_{0} z^{k}}\right)\right\} \\
& -\left\{n A_{n}(z)+\cdots+2 A_{2}(z)+A_{1}(z) e^{\alpha_{1} z^{k}}\right\} \not \equiv 0 .
\end{aligned}
$$

Since $\max \left\{\rho\left(A_{j}\right), \rho\left(B_{0}\right): 0 \leq j \leq n\right\}=\alpha<k$, we have $\rho(D) \leq k$.

Now, for any given $\epsilon>0$, applying Lemma 2.1 and Theorem 1.1, we can deduce that

$$
\begin{aligned}
m( & \left.r, \frac{1}{g(z)}\right) \\
= & m\left(r, \frac{A_{n}(z) g(z+n)+\cdots+A_{2}(z) g(z+2)+A_{1}(z) e^{\alpha_{2} z^{k}} g(z+1)+\left(A_{0}(z) e^{\alpha_{0} z^{k}}+B_{0}(z) e^{\beta_{0} z^{k}}\right) g(z)}{D(z) g(z)}\right) \\
\leq & \sum_{j=0}^{n} m\left(r, \frac{g(z+j)}{g(z)}\right)+\sum_{j=0}^{n} T\left(r, A_{j}(z)\right)+T\left(r, e^{\alpha_{1} z^{k}}\right)+T\left(r, B_{0}(z)\right) \\
& +T\left(r, e^{\alpha_{0} z^{k}}\right)+T\left(r, e^{\beta_{0} z^{k}}\right)+T(r, D(z))+O(\log r) \\
= & O\left(r^{\rho-1+\epsilon}\right)=S(r, g) .
\end{aligned}
$$

This implies that

$$
N\left(r, \frac{1}{f-z}\right)=N\left(r, \frac{1}{g}\right)=T(r, g)+S(r, g)=T(r, f)+S(r, f),
$$

then $\lambda(f-z)=\rho(f)$ follows.

Next, we assert that either $k+1 \leq \rho(f) \leq \max \left\{\lambda(f), \lambda\left(\frac{1}{f}\right)\right\}+1$ or $\rho(f)=k+1$. If the assertion does not hold, we have $\max \left\{k, \lambda(f), \lambda\left(\frac{1}{f}\right)\right\}+1<\rho(f)<\infty$.

Assume that $z=0$ is a zero (or a pole) of $f(z)$ of order $m$. Applying the Hadamard factorization of a meromorphic function, we write $f(z)$ as follows:

$$
f(z)=z^{m} \frac{P_{1}(z)}{P_{2}(z)} e^{Q(z)}
$$

where $P_{1}(z), P_{1}(z)$ are entire functions such that $\rho\left(P_{1}\right)=\lambda(f), \rho\left(P_{2}\right)=\lambda\left(\frac{1}{f}\right)$ and $Q(z)$ is a polynomial such that $\operatorname{deg} Q(z)=q>\max \left\{k, \lambda(f), \lambda\left(\frac{1}{f}\right)\right\}+1$.

Now, we obtain from (1.1) that

$$
\sum_{j=0}^{n} h_{j}(z) e^{Q(z+j)}=0,
$$


where

$$
h_{0}=\left(A_{0}(z) e^{\alpha_{0} z^{k}}+B_{0}(z) e^{\beta_{0} z^{k}}\right) z^{m} \frac{P_{1}(z)}{P_{2}(z)}, \quad h_{1}=A_{1}(z) e^{\alpha_{1} z^{k}}(z+1)^{m} \frac{P_{1}(z+1)}{P_{2}(z+1)},
$$

and

$$
h_{j}(z)=A_{j}(z)(z+j)^{m} \frac{P_{1}(z+j)}{P_{2}(z+j)} \quad(2 \leq j \leq n+1) .
$$

Notice that $\operatorname{deg}(Q(z+h)-Q(z+l))=q-1>\rho\left(h_{j}\right)$ for $0 \leq h<l \leq n$ and $0 \leq j \leq n$. Thus, Lemma 2.6 is valid for (3.20), hence we get that $h_{j}(z) \equiv 0$ for $j=1,2, \ldots, n+1$, a contradiction to our assumption. This completes our proof.

The proof of Theorem 1.4 is similar to that of Theorem 1.3.

\subsection{The proof of Theorem 1.5}

Let $f(\not \equiv 0)$ be a meromorphic solution of (1.4). Suppose that $\rho(f)<2$, then by Lemma 2.1, for any given $\epsilon>0$, there exists a subset $E \subset(1, \infty)$ of finite logarithmic measure such that for all $z$ satisfying $|z|=r \notin[0,1] \cup E$, and as $r$ sufficiently large, we have

$$
\left|\frac{f(z+j)}{f(z+l)}\right| \leq \exp \left\{r^{\rho(f)-1+\epsilon}\right\} \quad(j=0,1, \ldots, n, j \neq l)
$$

Set $z=r e^{i \theta}, \alpha_{j}=\left|\alpha_{j}\right| e^{i \varphi_{j}}$ and $\delta\left(\alpha_{j} z, \theta\right)=\left|\alpha_{j}\right| \cos \left(\varphi_{j}+\theta\right)(j=0,1, \ldots, k)$. Then $E_{1}=\{\theta \in[0,2 \pi)$ : $\left.\delta\left(\alpha_{j} z, \theta\right)=0, j=0,1, \ldots, n\right\} \cup\left\{\theta \in[0,2 \pi): \delta\left(\alpha_{j} z-\alpha_{i} z, \theta\right)=0,0 \leq i<j \leq n\right\}$ is a set of linear measure zero.

Consider that $a_{j}(z)=A_{j}(z) e^{\alpha_{j} z}, A_{j}(z)(\not \equiv 0)$ are meromorphic functions and $\rho\left(A_{j}\right)=\alpha<1$, by Lemma 2.5, for the above $\epsilon>0$, there exists a set $F_{j} \in[0,2 \pi)$ of linear measure zero such that for any $z=r e^{i \theta}$ satisfying $\theta \in[0,2 \pi) \backslash\left(E_{1} \cup F_{j}\right)$, and as $r \rightarrow \infty$, we have

(i) if $\delta\left(\alpha_{j} z, \theta\right)>0$, then

$$
\exp \left\{(1-\epsilon) \delta\left(\alpha_{j} z, \theta\right) r\right\} \leq\left|a_{j}\left(r e^{i \theta}\right)\right| \leq \exp \left\{(1+\epsilon) \delta\left(\alpha_{j} z, \theta\right) r\right\}
$$

(ii) if $\delta\left(\alpha_{j} z, \theta\right)<0$, then

$$
\exp \left\{(1+\epsilon) \delta\left(\alpha_{j} z, \theta\right) r\right\} \leq\left|a_{j}\left(r e^{i \theta}\right)\right| \leq \exp \left\{(1-\epsilon) \delta\left(\alpha_{j} z, \theta\right) r\right\}
$$

Set $E_{2}=\bigcup_{j=0}^{n} F_{j}$, then $E_{2}$ is a set of linear measure zero.

Since $\alpha_{j}$ are distinct complex constants, then there exists only one $l \in\{0,1, \ldots, n\}$ such that $\delta\left(\alpha_{l} z, \theta\right)=\max \left\{\delta\left(\alpha_{j} z, \theta\right): j=0,1, \ldots, n\right\}$ for any $\theta \in[0,2 \pi) \backslash\left(E_{1} \cup E_{2}\right)$. Now we take a ray $\arg z=\theta_{0} \in[0,2 \pi) \backslash\left(E_{1} \cup E_{2}\right)$ such that $\delta\left(\alpha_{l} z, \theta_{0}\right)>0$.

Let $\delta_{1}=\delta\left(\alpha_{l} z, \theta_{0}\right), \delta_{2}=\max \left\{\delta\left(\alpha_{j} z, \theta_{0}\right): j=0,1, \ldots, l, j \neq l\right\}$, then $\delta_{1}>\delta_{2}$. We discuss the following two cases.

Case 1. $\delta_{2}>0$. We rewrite (1.4) in the form

$$
-a_{l}(z)=\sum_{j=0}^{n} a_{j}(z) \frac{f(z+j)}{f(z+l)} \quad(j \neq l) .
$$


By (3.21), (3.22) and (3.24), we get for $z=r e^{i \theta_{0}}$ and sufficiently large $r \notin[0,1] \cup E$,

$$
\begin{aligned}
\exp \left\{(1-\epsilon) \delta_{1} r\right\} & \leq\left|a_{l}\left(r e^{i \theta_{0}}\right)\right| \leq \sum_{j=0, j \neq l}^{n} \exp \left\{(1+\epsilon) \delta\left(\alpha_{j} z, \theta\right) r\right\} \exp \left\{r^{\rho(f)-1+\epsilon}\right\} \\
& \leq n \exp \left\{(1+\epsilon) \delta_{2} r\right\} \exp \left\{r^{\rho(f)-1+\epsilon}\right\}
\end{aligned}
$$

When $0<2 \epsilon<\min \left\{\frac{\delta_{1}-\delta_{2}}{\delta_{1}+\delta_{2}}, 2-\rho(f)\right\}$, by (3.25), we get

$$
\exp \left\{\frac{\delta_{1}-\delta_{2}}{2} r\right\} \leq n \exp \left\{r^{\rho(f)-1+\epsilon}\right\}
$$

This is impossible.

Case 2. $\delta_{2}<0$. By (3.21), (3.23) and (3.24), we get for $z=r e^{i \theta_{0}}$ and sufficiently large $r \notin$ $[0,1] \cup E$,

$$
\begin{aligned}
\exp \left\{(1-\epsilon) \delta_{1} r\right\} & \leq\left|a_{l}\left(r e^{i \theta_{0}}\right)\right| \leq \sum_{j=0, j \neq l}^{n} \exp \left\{(1-\epsilon) \delta\left(\alpha_{j} z, \theta\right) r\right\} \exp \left\{r^{\rho(f)-1+\epsilon}\right\} \\
& \leq n \exp \left\{r^{\rho(f)-1+\epsilon}\right\} .
\end{aligned}
$$

This is a contradiction. Hence we get $\rho(f) \geq 2$.

\subsection{The proof of Theorem 1.6}

Considering $a_{j}(z)=A_{j}(z) e^{\alpha_{j} z}+D_{j}(z)(j=0,1, \ldots, n), A_{j}(z)(\not \equiv 0), D_{j}(z)(\not \equiv 0)$ are meromorphic functions and $\rho\left(A_{j}\right)=\alpha<1, \rho\left(D_{j}\right)=\beta<1$, by Lemmas 2.2 and 2.5, we know that for any given $\epsilon>0$, there exists a subset $E \subset(1, \infty)$ of finite logarithmic measure such that, for all $z$ satisfying $|z|=r \notin[0,1] \cup E$, and as $r \rightarrow \infty$, we have

(i) if $\delta\left(\alpha_{j} z, \theta\right)>0$, then

$$
\begin{aligned}
\exp & \left\{(1-\epsilon) \delta\left(\alpha_{j} z, \theta\right) r\right\}-\exp \left\{r^{\beta+\epsilon}\right\} \\
& <\left|a_{j}\left(r e^{i \theta}\right)\right|<\exp \left\{(1+\epsilon) \delta\left(\alpha_{j} z, \theta\right) r\right\}+\exp \left\{r^{\beta+\epsilon}\right\} \\
& <(1+o(1)) \exp \left\{(1+\epsilon) \delta\left(\alpha_{j} z, \theta\right) r\right\} ;
\end{aligned}
$$

(ii) if $\delta\left(\alpha_{j} z, \theta\right)<0$, then

$$
\left|a_{j}\left(r e^{i \theta}\right)\right|<\exp \left\{(1-\epsilon) \delta\left(\alpha_{j} z, \theta\right) r\right\}+\exp \left\{r^{\beta+\epsilon}\right\}<(1+o(1)) \exp \left\{r^{\beta+\epsilon}\right\} .
$$

Then, using a similar argument to that of Theorem 1.5 and Theorem 1.1 and only replacing (3.22) (or (3.23)) by (3.26) (or (3.27)), we can prove Theorem 1.6.

\subsection{The proof of Theorem 1.7}

Let $f(z) \not \equiv 0$ be a meromorphic solution of (1.5). Suppose that $\rho(f)<1$, then by Lemma 2.7, we obtain $\rho(F)=\rho\left(\sum_{j=0}^{k} a_{j}(z) f(z+j)\right)=1$. This contradicts $\rho(F)<1$, therefore we have $\rho(f) \geq 1$.

Suppose that there exist two distinct meromorphic solutions $f_{1} \not \equiv 0, f_{2} \not \equiv 0$ of Eq. (1.5) such that $\max \left\{\rho\left(f_{1}\right), \rho\left(f_{2}\right)\right\}<2$, then $f_{1}-f_{2}$ is a meromorphic solution of the homogeneous 
linear difference equation corresponding to (1.5) and $\rho\left(f_{1}-f_{2}\right)<2$. By Theorem 1.5 or Theorem 1.6, we get a contradiction. So Eq. (1.5) has at most one meromorphic solution $f_{0}$ satisfying $1 \leq \rho\left(f_{0}\right) \leq 2$.

Next we prove $\max \left\{\lambda\left(f_{0}\right), \lambda\left(\frac{1}{f_{0}}\right)\right\}=\rho\left(f_{0}\right)$ in the case $\rho\left(f_{0}\right)=1$. Suppose that $\max \left\{\lambda\left(f_{0}\right)\right.$, $\left.\lambda\left(\frac{1}{f_{0}}\right)\right\}<\rho\left(f_{0}\right)$, then by the Weierstrass factorization, we obtain

$$
f_{0}(z)=\frac{P_{1}(z)}{P_{2}(z)} e^{Q(z)}
$$

where $P_{1}(z), P_{1}(z)$ are entire functions such that $\rho\left(P_{1}\right)=\lambda\left(P_{1}\right)=\lambda\left(f_{0}\right), \rho\left(P_{2}\right)=\lambda\left(P_{2}\right)=\lambda\left(\frac{1}{f_{0}}\right)$ and $Q(z)$ is a polynomial of degree 1 .

In the case $a_{j}(z)=A_{j}(z) e^{\alpha_{j} z}$. Substituting (3.28) into (1.5), we get

$$
\sum_{j=0}^{n} A_{j}(z) \frac{P_{1}(z+j)}{P_{2}(z+j)} e^{\alpha_{j} z+Q(z+j)}=F(z) .
$$

Since $\alpha_{j}$ are distinct complex numbers, by Lemma 2.7, we obtain that the order of the left-hand side of (3.29) is 1 . This contradicts $\rho(F)<1$.

For $a_{j}(z)=A_{j}(z) e^{\alpha_{j} z}+D_{j}(z)$, by using an argument similar to the above, we also obtain a contradiction.

It is obvious that $\max \left\{\lambda\left(f_{0}\right), \lambda\left(\frac{1}{f_{0}}\right)\right\}=\rho\left(f_{0}\right)$ provided that $1<\rho\left(f_{0}\right)<2$. Therefore we have $\max \left\{\lambda\left(f_{0}\right), \lambda\left(\frac{1}{f_{0}}\right)\right\}=\rho\left(f_{0}\right)$.

\section{Competing interests}

The authors declare that they have no competing interests.

\section{Authors' contributions}

ZLY performed and drafted manuscript. All authors read and approved the final manuscript.

\section{Acknowledgements}

The author thanks the referee for his/her valuable suggestions to improve the present article. The research was supported by the Beijing Natural Science Foundation (No. 1132013) and the Foundation of Beijing University of Technology (No. 006000514313002).

Received: 9 July 2014 Accepted: 14 November 2014 Published: 03 Dec 2014

\section{References}

1. Hayman, WK: Meromorphic Functions. Claredon Press, Oxford (1964)

2. Laine, I: Nevanlinna Theory and Complex Differential Equations. de Gruyter, Berlin (1993)

3. Yang, CC, Yi, HX: Uniqueness Theory of Meromorphic Functions. Kluwer Academic, Dordrecht (2003)

4. Zhang, J: Some results on zeros and the uniqueness of one certain type of high difference polynomials. Adv. Differ. Equ. 2012, Article ID 160 (2012)

5. Chen, ZX: Growth and zeros of meromorphic solution of some linear difference equations. J. Math. Anal. Appl. 373, 235-241 (2011)

6. Ishizaki, K, Yanagihara, N: Wiman-Valiron method for difference equations. Nagoya Math. J. 175, 75-102 (2004)

7. Laine, I: Nevanlinna Theory and Complex Differential Equations. de Gruyter, Berlin (1993)

8. Li, S, Gao, ZS: Finite order meromorphic solutions of linear difference equations. Proc. Jpn. Acad., Ser. A, Math. Sci. 87(5), 73-76 (2011)

9. Zheng, XM, Tu, J: Growth of meromorphic solutions of linear difference equations. J. Math. Anal. Appl. 384, 349-356 (2011)

10. Chen, ZX: The growth of solutions of a class of second-order differential equations with entire coefficients. Chin. Ann Math., Ser. B 20(1), 7-14 (1999) (in Chinese)

11. Liu, Y: On growth of meromorphic solutions for linear difference equations with meromorphic coefficients. Adv Differ. Equ. 2013, Article ID 60 (2013)

12. Laine, I, Yang, CC: Clunie theorems for difference and q-difference polynomials. J. Lond. Math. Soc. 76(2), 556-566 (2007)

13. Chiang, YM, Feng, SJ: On the Nevanlinna characteristic $f(z+\eta)$ and difference equations in the complex plane. Ramanujan J. 16, 105-129 (2008) 
14. Chiang, YM, Feng, SJ: On the growth of logarithmic differences, difference quotients and logarithmic derivatives of meromorphic functions. Trans. Am. Math. Soc. 361(7), 3767-3791 (2009)

15. Peng, F, Chen, ZX: On the growth of solutions of some second-order linear differential equations. J. Inequal. Appl. 2011, Article ID 635604 (2011)

16. $\mathrm{Xu}, \mathrm{J}$, Zhang, $\mathrm{X}$ : Some results of meromorphic solutions of second-order linear differential equations. J. Inequal. Appl. 2013, Article ID 304 (2013)

17. Li, S, Chen, B: Results on meromorphic solutions of linear difference equations. Adv. Differ. Equ. 2012, Article ID 203 (2012)

18. Liu, H, Mao, Z: On the meromorphic solutions of some linear difference equations. Adv. Differ. Equ. 2013, Article ID $133(2013)$

19. Chen, ZX, Shon, $\mathrm{KH}$ : On the growth and fixed points of solutions of second order differential equation with meromorphic coefficients. Acta Math. Sin. Engl. Ser. 21(4), 753-764 (2005)

20. Halburd, RG, Korhonen, R: Finite-order meromorphic solutions and the discrete Painlevé equations. Proc. Lond. Math. Soc. 94, 443-474 (2007)

10.1186/1687-1847-2014-306

Cite this article as: Yuan and Ling: Results on the growth of meromorphic solutions of some linear difference equations with meromorphic coefficients. Advances in Difference Equations 2014, 2014:306

\section{Submit your manuscript to a SpringerOpen ${ }^{\circ}$ journal and benefit from:}

- Convenient online submission

Rigorous peer review

- Immediate publication on acceptance

- Open access: articles freely available online

- High visibility within the field

- Retaining the copyright to your article 